\title{
An Intrinsically Magnetic Topological Insulator
}

\author{
Experiments show that the ferromagnetism naturally possessed by the \\ topological insulator manganese bismuth telluride extends right to the \\ material's surface.
}

By Erika K. Carlson

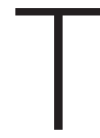

opological insulators that are intrinsically magnetic - rather than made to have a field by the incorporation of magnetic impurities-may provide opportunities to study how magnetism affects these materials' distinctive electron-transport properties. Researchers have recently shown that the topological insulator manganese bismuth telluride $\left(\mathrm{MnBi}_{2} \mathrm{Te}_{4}\right)$ is naturally magnetically ordered in its interior, but they had not determined whether this bulk ferromagnetism extends to the surface. Now, Dan Nevola of Brookhaven National Laboratory, New York, and colleagues have experimentally confirmed that $\mathrm{MnBi}_{2} \mathrm{Te}_{4}$ is magnetically ordered at its surface as well [1].

Nevola and colleagues used photoemission spectroscopy to try to spot the effects of magnetism on the energy bands of electrons at the surface of an $\mathrm{MnBi}_{2} \mathrm{Te}_{4}$ crystal. The researchers looked at two particular features of the surface band structure-a Dirac cone and a Rashba state. They found that an

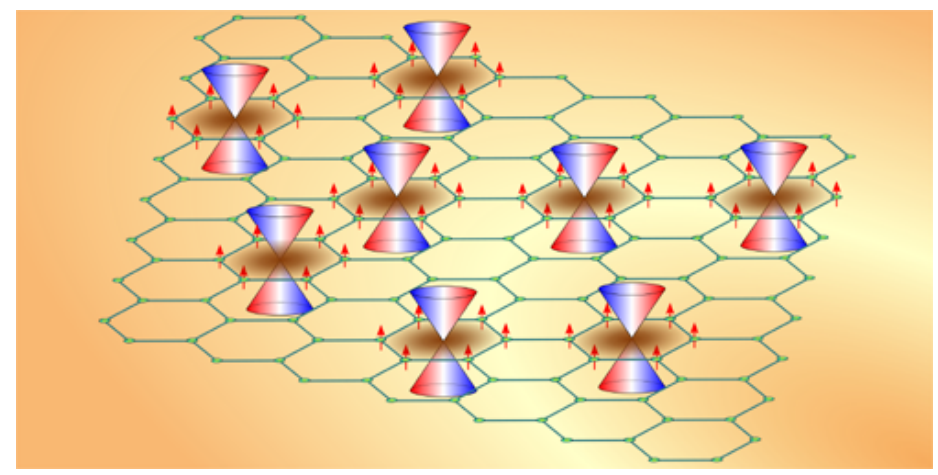

Credit: D. Nevola/Brookhaven National Laboratory energy gap was present in the Rashba state but, contrary to some predictions, no such gap existed in the Dirac cone.

Incorporating ferromagnetic order at the surface of the crystal into theoretical models reproduced the same energy gap in the Rashba state that the researchers observed in the experiments. Thus the crystal must have a magnetically ordered surface. The fact that the Dirac cone didn't behave as expected indicates that there is further complexity to be understood in this strange material.

The finding reveals a more complex view of intrinsic magnetic topological insulators in general, and of $\mathrm{MnBi}_{2} \mathrm{Te}_{4}$ specifically. In particular, the researchers say that it may provide insights into some of this material's mysterious properties, such as the especially low temperature at which it exhibits the quantum anomalous Hall effect.

Erika K. Carlson is a Corresponding Editor for Physics based in New York City.

\section{REFERENCES}

1. D. Nevola et al., "Coexistence of surface ferromagnetism and a gapless topological state in $\mathrm{MnBi}_{2} \mathrm{Te}_{4}$," Phys. Rev. Lett. 125, 117205 (2020). 